\title{
La tarea escolar: representaciones lingüísticas de los estudiantes
}

\author{
Homework: Linguistic Representations of the Students
}

\section{A lição de casa: representações linguísticas dos estudantes}

\author{
Jaime Ruiz Vega \\ Álvaro William Santiago Galvis \\ Myriam Castillo Perilla ${ }^{1}$
}

\section{Resumen}

El presente artículo da cuenta de algunos de los resultados que arrojó el desarrollo del proyecto de investigación Concepciones de los estudiantes de educación básica secundaria y media de Bogotá sobre la entidad léxica tarea escolar, DLE-427-16, que tuvo como objetivo establecer y analizar las representaciones y los valores sociales que de la entidad léxica tarea escolar tienen los estudiantes de la educación básica secundaria y media de Bogotá. Este trabajo fue orientado por los principios teóricos y metodológicos de la Semántica de los Posibles Argumentativos (SPA); fundamentalmente el supuesto que plantea que en la acción discursiva, y en especial en las formas léxicas que la constituyen, los hablantes imprimen una axiologización del mundo, en razón a que dichas formas tienen un potencial argumentativo. En este sentido, se logró reconstruir el núcleo o elementos fundamentales del significado de esa entidad léxica, así como los estereotipos sobre la base de las definiciones redactadas por los informantes. Se identificaron y estudiaron los valores sociales complejos que la población de la investigación activó en sus concepciones y en sus definiciones. Para dar cuenta de ello, en primer lugar, se hace una presentación de los referentes conceptuales de la SPA; luego las consideraciones de orden metodológico y, finalmente, se presenta la discusión de los datos obtenidos, en lo que respecta a la reconstrucción del núcleo y la identificación de los estereotipos.

\section{Palabras clave}

significado léxico; tarea escolar; núcleo; estereotipos;valores sociales; representación lingüística

\section{Abstract}

This paper reports on some of the results of the research project “Conceptions of Secondary and Middle School Students in Bogotá about the Lexical Entity Homework," DLE-427-16, which sought to establish and analyze the representations and social values that secondary and middle school students in Bogota have about lexical entity homework. This work was guided by the theoretical and methodological principles of Semantics of Possible Arguments (SPA), mainly on the hypothesis that, in discursive action, and especially in the lexical forms that constitute it, speakers express an axiologization of the world, since these forms have an argumentative potential. In this sense, we were able to reconstruct the core or fundamental elements of the meaning of that lexical entity, as well as the stereotypes based on the definitions written by the informants. We identified and studied the complex social values that the research population activated in their conceptions and definitions. To account for this, first we present the conceptual referents of the SPA; then, the methodological considerations, and, finally, we discuss the data obtained, with regard to the reconstruction of the nucleus and the identification of stereotypes.

\section{Keywords}

lexical meaning; homework; core; stereotypes; social values; linguistic representation

1 Universidad Pedagógica Nacional, Bogotá, Colombia. Correos electrónicos: jruiz@pedagogica.edu.co; asantiago@pedagogica.edu.co; mcastillo@pedagogica.edu.co. Integrantes del Grupo de Investigación en Pedagogía, Lenguaje y Comunicación (Gipelec). 


\section{Resumo}

0 presente artigo apresenta algumas das descobertas obtidas no desenvolvimento do projeto de pesquisa Concepções dos estudantes de educação básica secundária e média em Bogotá sobre a entidade léxica lição de casa, DLE-427-16, que visou estabelecer e analisar as representações e os valores sociales que os estudantes de Educação Básica e Média de Bogotá têm sobre a entidade léxica lição de casa. Este trabalho baseou-se nos princípios teóricos e metodológicos da Semântica dos Possíveis Argumentos (SPA), fundamentalmente, na hipótese de que, na ação discursiva, especialmente nas formas léxicas que a constituem, os falantes plasmam uma axiologização do mundo, devido a que essas formas têm um potencial argumentativo. Neste sentido, foi possível reconstruir o núcleo ou elementos fundamentais do significado desta entidade lexical, assim como os estereótipos sobre a base das definições redigidas pelos informantes. Os valores sociais complexos que a população da pesquisa ativou em suas concepções e definições foram estudados e analisados. Para isso, em primeiro lugar, apresentam-se os referentes conceituais da SPA; em segundo lugar, as considerações metodológicas e, finalmente, a discussão dos dados obtidos em relação com a reconstrução do núcleo e a identificação dos estereótipos.

\section{Palavras-chave}

significado léxico; lição de casa; núcleo; estereótipos; valores sociais; representação linguística

\section{Artículo recibido el 11 de mayo de 2017 y aprobado el 10 de enero de 2018}

\section{Introducción}

Los llamados deberes escolares, i. e., las tareas escolares, se constituyen, en principio, como puente que vincula las prácticas que se dan en el ámbito escolar y el contexto, esto es, el mundo aparentemente externo a la institución educativa. Estos deberes, hace ya un tiempo, se han tornado en tema y actividad objeto de preocupación, de discusión, de propuestas y de decisiones en los órdenes nacional e internacional. Asignadas y calificadas por los profesores, desarrolladas por estudiantes y acudientes o solamente por estos últimos, y caracterizadas por múltiples aristas, las tareas escolares son el blanco de diversas críticas de distintos actores educativos, a tal punto que con frecuencia se considera que carecen de una programación, de un seguimiento y valoración, de una incidencia positiva y de un mejoramiento permanente. En pocas palabras, las prácticas ligadas a las tareas, y las tareas en sí, son cuestionadas progresivamente.

De acuerdo con lo anterior, el trabajo que aquí se presenta aborda la problemática de las tareas escolares, desde la óptica de uno de los protagonistas del proceso de enseñanza/aprendizaje: los estudiantes. En este orden de ideas, este artículo expone parte de los resultados que se obtuvieron del desarrollo del proyecto de investigación "Concepciones de los estudiantes de educación básica secundaria y media de Bogotá sobre la entidad léxica tarea escolar, DLE427-16", que tuvo como finalidad la identificación y el análisis de las representaciones y los valores sociales de los estudiantes de educación básica secundaria y media de Bogotá, específicamente de los ciclos 3,4 y 5, sobre la entidad léxica ${ }^{2}$ tarea escolar. La labor se adelantó a la luz de los desarrollos teóricos y metodológicos de la semántica de los posibles argumentativos (SPA).

\section{Fundamentos conceptuales de la SPA}

El análisis del discurso es un terreno favorable para dar cuenta de la actividad humana y, en este marco, para estudiar el sentido que los interlocutores, los sujetos sociales, dan a sus acciones y las dinámicas de identidad (Galatanu, 2000). Entonces, es en, y por, el discurso que el sujeto lleva a cabo una valoración, una ruptura de la indiferencia, esto es, una axiologización de sí mismo, de los otros sujetos, de sus interacciones y, en general, de los objetos y relaciones que existen en el mundo natural y cultural.

Denominada inicialmente como semántica argumentativa integrada (Galatanu, 1999, 2000), la semántica de los posibles argumentativos (SPA), en la línea de la semántica argumentativa, forja y propone un modelo de descripción del significado léxico capaz de dar cuenta tanto de la manera como en la lengua se percibe y, a su vez, se moldea el mundo (Galatanu, 2003a), como del potencial argumentativo de las palabras. En este sentido, la sPA propone

2 En el ámbito de la SPA se denomina entidad léxica a las formas modales, palabras, expresiones que son objeto de estudio. En este artículo se recurre a este término y a forma modal, palabra, expresión, término o forma léxica. 
un protocolo para analizar las representaciones lingüísticas y la axiologización de la realidad portada por las entidades léxicas a las que recurre el sujeto para establecerse en el mundo, interactuar en él y dar cuenta de esa realidad.

De otro lado, la SPA se orienta por las propuestas teóricas sobre los estereotipos lingüísticos, de tal forma que Galatanu (2003a), a partir de los planteamientos de Putnam (1975) sobre el significado de las entidades léxicas ${ }^{3}$, propone un modelo de análisis del significado léxico estructurado en tres estratos: el núcleo, los estereotipos y los posibles argumentativos. A estos tres estratos, Galatanu (2009) agrega una forma de manifestación discursiva: los despliegues argumentativos, que sitúan el modelo en la interface de la lengua y del discurso\{XE “discours"\}.

El núcleo de la entidad léxica, primer estrato, remite a los rasgos de categorización semántica $\{\mathrm{XE}$ "sémantique" $\}$ de Putnam (1975) como el elemento estable del significado léxico $\{\mathrm{XE}$ "signification lexicale" $\}$. E $\{\mathrm{XE}$ "SPA" $\}$ stos rasgos o propiedades esenciales de la palabra, en la orientación de la SPA, se describen en términos de predicados abstractos y corresponden a primitivos semánticos. Los estereotipos corresponden a $\{\mathrm{XE}$ " $S P A$ " $\}$ "un conjunto abierto de elementos del núcleo con otras representaciones y que constituyen bloques de argumentación $\{\mathrm{XE}$ “argumentation"\} interna” (Galatanu, 2009, p. 395), y se describen en términos de bloques de significado argumentativo que establecen relaciones entre elementos del núcleo y otras representaciones semánticas (Galatanu, 2006).

Entre tanto, los posibles argumentativos constituyen secuencias discursivas estructuradas en bloques de argumentación externa, gracias a un cálculo realizado por el investigador que se ocupa del estudio del potencial argumentativo de las entidades léxicas. Se calculan estas secuencias a partir de la asociación de la unidad lingüística (objeto de estudio)\{XE "entitélexicale" $\}$ con uno de los elementos del conjunto amplio y abierto de estereotipo $\{\mathrm{XE}$ “stéréotype:stéréotypes”\}s.

3 Este filósofo define el significado de las palabras en términos de núcleo de rasgos de categorización y de estereotipos asociados a la palabra de manera duradera.
Se trata, entonces, de asociaciones virtuales que "se organizan en dos haces orientados respectivamente hacia uno o el otro de los polos axiológicos $\{\mathrm{XE}$ “axiologiques" $\}$ (positivo y negativo)" (Galatanu, 2005, p. 57). El entorno semántico\{XE “sémantique”\} o el contexto $\{\mathrm{XE}$ “contexte" $\}$ cumplen una función importante en los posibles argumentativos en razón a que pueden producir una contaminación discursiva que determina, a su vez, la orientación positiva o negativa del haz de asociaciones. A partir de esta organización estructurada en dos haces, los posibles argumentativos pueden ser conformes o no conformes a la orientación axiológica del estereotipo, $\{\mathrm{XE}$ "stéréotype:stéréotypes"\} esto debido a que incluso en el núcleo, o en los estereotipos de la palabra, el potencial axiológico es doble (positivo y negativo a la vez).

Por su parte, los despliegues argumentativos, o secuencias argumentativas ejecutadas en las ocurrencias discursivas, son una forma de manifestación discursiva o "secuencias argumentativas realizadas por las ocurrencias discursivas" (Galatanu, 2009, p. 395). Este nivel del modelo de representación existe efectivamente en el discurso\{XE "discours" y permite estudiar la actualización de los posibles argumentativos. "Es la puesta en el discurso [la aparición en el discurso] de las entidades léxicas y sus interacciones [en este] lo que hace aparecer los despliegues argumentativos" (Ruiz, 2013, p. 36), lo que presenta al discurso como un fenómeno observable fundamental en la SPA, por tanto, privilegiado por esta orientación de la semántica argumentativa. Gracias al análisis de estas secuencias argumentativas es posible establecer las relaciones que se generan entre el sentido\{XE "sens" $\}$ de las ocurrencias discursivas y el significado de la unidad léxica. Asimismo, permite verificar, posteriormente, la conformidad o la no conformidad del sentido discursivo generado con el protocolo semántico $\{\mathrm{XE}$ "sémantique"\} del significado.

Con miras a obtener de manera apropiada la representación semántica de las entidades léxicas, la SPA $\{\mathrm{XE}$ "SPA" $\}$ determina como indispensables las cuatro condiciones siguientes (Galatanu, 2007, p. 316): 
- Prever y exponer $\{\mathrm{XE}$ “représentation” $\}$ una dimensión descriptiva del significado léxico $\{\mathrm{XE}$ "signification lexicale"\}.

- Dar cuenta del núcleo, esto es, del componente estable del significado, así como de la dimensión evolutiva en la que se activan o desactivan valores una vez que el término aparece en discurso\{XE “discours" $\}$ XE "valeurs" $\}$.

- Identificar la característica de conjunto abierto de elementos de significado evolutivos, lo que corresponde a los estereotipos [lingüísticos].

- Precisar el potencial discursivo, es decir, la dimensión argumentativa del significado léxico\{XE "signification lexicale"\}.

En ese sentido, el investigador deberá recurrir a los diccionarios de una lengua, los más representativos o los de uso más frecuente, para determinar la representación semántica de las palabras que son su objeto de estudio. Desde las definiciones lexicográficas deberá, entonces, identificar el núcleo, por una parte; y a partir de ejemplos, citas y usos de los términos en cuestión podrá dar cuenta del conjunto abierto de representaciones o estereotipos. Una vez identificados estos dos estratos tendrá los elementos para calcular los posibles argumentativos.

Sumado a lo expuesto se debe precisar que lejos de buscar determinar el carácter verdadero o falso de las palabras, el estudioso se propone establecer cómo el sujeto (se) representa el mundo en el que existe y las relaciones que se tejen entre los sujetos. Por tanto, "el análisis del discurso $\{\mathrm{XE}$ "discours" $\}$ no se enfoca en saber si tal o cual enunciado es verídico, sino que se interesa ante todo en las representaciones que están presentes en el discurso" (Dervin, 2009, p. 170). Mediante la lengua, el hablante no solamente describe el mundo y narra lo que en él sucede, sino que -en especial- refleja una ruptura de la indiferencia frente a esa realidad. En ese mismo sentido, (se) representa el mundo, porta valoraciones y asume actitudes sobre esa realidad. De esa manera, imprime en las unidades lingüísticas de su discurso una axiologización y ello porque estas están dotadas de un potencial argumentativo, y ejecuta entonces "evaluaciones, ligadas, referidas a campos de experiencia humana" (Galatanu, 2003a, p. 217). Estas actividades del hablante toman forma en y por el discurso\{XE “discours” . Al respecto, Galatanu (2003b) propone una hipótesis según la cual

El discurso $\{\mathrm{XE}$ “discours” $\}$, aunque no tenga como objeto los valores, los problemas de ética, de moral, de deontología, o como objetivo la transmisión, el refuerzo o también la reconstrucción \{XE “reconstruction" $\}$ de un sistema de valores, no es menos evaluativo de la representación $\{\mathrm{XE}$ “représentation"\} que él propone del mundo y, por consiguiente, portador de valores. (p. 87).

En consecuencia, el análisis del discurso $\{\mathrm{XE}$ "discours"\} contribuye a abordar los discursos que se proponen estudiar, transmitir, confortar e incluso preservar y adquirir valores, así como a estudiar las orientaciones edificantes que pueden aparecer en diferentes tipos de discurso. A estas dos posibilidades se debe agregar una referida a la dimensión argumentativa de los discursos y que al estar " $\{\mathrm{XE}$ "discours" inscrita en el significado de las unidades léxicas utilizadas o construida en contexto $\{\mathrm{XE}$ “contexte" , autoriza una orientación axiológica (negativa o positiva) de los encadenamientos discursivos" $\{\mathrm{XE}$ "enchaînements discursisfs" (Galatanu, 2003b, p. 88)

$\mathrm{Al}$ ocuparse de esta ruptura de la indiferencia, esto es, de la presencia de valores portados por las entidades léxicas y activados en las ocurrencias discursivas, $\{\mathrm{XE}$ "linguistique" $\}$ la SPA $\{\mathrm{XE}$ "SPA" $\}$ recurre a los conceptos $\{\mathrm{XE}$ “discours" $\}$ modalización, modalidad y valor modal. Galatanu (2002) define la modalización como

[...] la inscripción en el enunciado, mediante una marca (huella) lingüística (formas modales), de la actitud (valor modal) [intelectual, volitiva, evaluativa] del sujeto comunicante respecto del contenido proposicional de su enunciado y de la función que este enunciado está llamado a tener en la interacción verbal en la que el sujeto participa. (p. 20).

Por otra parte, la actitud del hablante aparece, queda marcada, en las unidades lingüísticas a que 
él recurre, esto es, la modalidad. Por último, esa actitud del hablante, presente en las formas léxicas activadas, corresponde a su actitud hacia el "contenido proposicional de su enunciado y de la función que este enunciado cumple aparentemente en la interacción verbal en la que participa”. (Galatanu, 2003b, p. 90)\{XE “modalisation”\} \{XE “forme modale: formes modales" $\}$.

Tanto las formas modales como los valores modales corresponden a elementos fundamentales en el estudio realizado sobre la tarea escolar. Se busca identificar, por un lado, las asociaciones y definiciones de esta entidad léxica, que se plasman en las formas modales, y, del otro, identificar los valores modales que contienen. Galatanu (2003b) afirma que estos últimos se materializan en las formas modales activadas en el discurso \{XE "discours" $\}$ del sujeto comunicante. Estas actitudes o valoraciones, u orientaciones axiológicas, contenidas en las formas modales de las manifestaciones discursivas del hablante, se estructuran \{XE “locuteur" $\}$ en cuatro zonas semánticas modales \{XE “zones sémantiques modales” $\}$ : zona modal $\{\mathrm{XE}$ "zone modale" $\}$ ontológica, zona modal $\{\mathrm{XE}$ "zone modale" $\}$ de juicio de verdad, zona modal $\{\mathrm{XE}$ "zone modale" $\}$ axiológica y zona modal $\{\mathrm{XE}$ "zone moda$l e "\}$ de los valores finalizantes.

De acuerdo con Ruiz, Castillo y Santiago (2016, p. 157), en la zona modal ontológica se sitúan los valores aléticos y los deónticos. Estos dos tipos de valores conciernen a valores que remiten a la existencia del mundo natural y social, y ello en y por el discurso. La comprensión del funcionamiento de las leyes naturales se manifiesta en los valores aléticos, los cuales se organizan sobre las ideas de la necesidad, la imposibilidad, la posibilidad y lo aleatorio. El mundo social y cultural y sus reglas son referidos por valores deónticos que se estructuran en torno a la obligación/obligatoriedad, la prohibición, lo permitido y lo opcional.

La zona semántica de los valores juicios de verdad alude a saberes/conocimientos y a creencias. Contenidos en esta zona modal, los valores epistémicos dan cuenta de lo seguro/certero/cierto, de lo incierto, de lo probable y de lo improbable, y se refieren al saber, al conocimiento y a la certeza que de ello tiene el sujeto. Las creencias de los hablantes están vinculadas a los valores doxológicos y estos últimos aparecen en formas verbales creer/no creer.

La tercera zona semántica modal, o zona modal axiológica, corresponde a lo que se denomina a menudo sistema de valores (Galatanu, 2003b), es decir, aquello que considera bueno, lo práctico, lo bonito, lo inútil, lo desagradable, lo interesante, etc. Estos valores portan las evaluaciones, las percepciones del ser humano sobre sus experiencias, su existencia en el mundo, sus interacciones. Por tanto, marcan, de manera decidida, una orientación hacia la preferencia, la ruptura, la elección, la supresión o debilitamiento de la indiferencia del sujeto frente a lo que (le) sucede en el mundo.

La última zona modal, que corresponde a los valores finalizantes, denominados también como de juicio de intención, da cuenta de la voluntad y del deseo de los sujetos. Forman parte de esta zona los valores volitivos (intención, voluntad) y los desiderativos (deseo, desear). Al situar estas zonas modales en el eje evaluativo presente en la praxis del sujeto, la zona ontológica existe en el polo objetivo, mientras los finalizantes, en el polo subjetivo.

A modo de cierre, los aspectos teóricos expuestos soportan el estudio de los datos recogidos con miras a analizar las representaciones lingüísticas que los sujetos encuestados tienen de la entidad léxica tarea escolar, así como a dar cuenta de los valores sociales complejos portados por las diversas formas modales inscritas en sus respuestas. Tanto el núcleo reconstruido como los estereotipos que son asociados a tarea escolar se clarifican y estudian a la luz de la propuesta teórica aquí sintetizada.

\section{Aspectos metodológicos}

En términos metodológicos, el trabajo se llevó a cabo bajo los preceptos de la SPA, que establece un protocolo de investigación que se estructura sobre un fundamento metodológico investigativo mixto, que implica, la recolección de datos, su respectiva cuantificación y el análisis cualitativo de las 
representaciones activadas y de los valores movilizados en las respuestas de los informantes.

Desde la perspectiva de la SPA, el análisis semántico corresponde a la construcción del objeto teórico denominado significado léxico (Galatanu, 2006, p. 132), que se elabora a partir de hipótesis externas sobre el sentido de las ocurrencias en la movilización de las entidades léxicas. Para esto, se han establecido dos grandes etapas: construcción y reconstrucción del significado léxico de la unidad léxica objeto de análisis.

La primera etapa se lleva a cabo partir del discurso lexicográfico, de ahí que se tome como referencia los diccionarios, específicamente, diccionarios representativos de la lengua española (DRAE; Diccionario de uso del español, de María Moliner) ${ }^{4}$. Esta construcción del significado brinda la posibilidad de establecer el núcleo, los estereotipos y los posibles argumentativos de la entidad léxica tarea (escolar), a partir del discurso codificado en los citados diccionarios.

La segunda etapa tiene que ver con la identificación de las ocurrencias relacionadas con tarea, que se evidencian en un grupo en particular, para este caso, estudiantes de educación básica y media de Bogotá. En esta etapa se da cuenta tanto de las representaciones léxicas como de la valoración activada en ellas, así como del cinetismo del significado léxico identificado en el discurso de la población participante en el estudio.

Dicha población estuvo conformada por 712 estudiantes de instituciones educativas de carácter público de la ciudad de Bogotá, ubicados en los ciclos $3^{\circ}, 4^{\circ}$ y $5^{\circ}$, de acuerdo con la organización del

4 En relación con esta etapa, se debe señalar que de ella se dio cuenta en el marco del proyecto de investigación "La movilización discursiva de valores sociales asociados a la entidad léxica tarea escolar en la educación básica y media en Bogotá, DLE-361-13", financiado por el Centro de Investigaciones de la Universidad Pedagógica Nacional, en el que se construyó el significado léxico de tarea escolar, se estableció el núcleo y los estereotipos con sus respectivos valores. Los dic cionarios citados se asumieron como los más representativos en Colombia, especialmente porque no se encontraron otros que presenten definiciones más completas $y$, además, porque en general las editoriales recurren a las propuestas del DRAE y de la obra de María Moliner. sistema escolar colombiano, distribuidos como se detalla en la tabla 1.

Tabla 1. Distribución de la población

\begin{tabular}{|l|l|l|}
\hline Ciclo & \multicolumn{1}{|c|}{ Grados } & \multicolumn{1}{c|}{ No. de estudiantes } \\
\hline \multirow{2}{*}{3} & Sexto & 120 \\
\cline { 2 - 3 } & Séptimo & 127 \\
\hline \multirow{2}{*}{4} & Octavo & 121 \\
\cline { 2 - 3 } & Noveno & 119 \\
\hline \multirow{2}{*}{5} & Décimo & 89 \\
\cline { 2 - 3 } & Undécimo & 136 \\
\hline
\end{tabular}

Fuente: elaboración propia.

Para efectos de obtener la información, se recurrió al cuestionario ${ }^{5}$ como instrumento, el cual se diseñó para identificar el núcleo, los estereotipos y la orientación axiológica presente en las ocurrencias. Para dar cuenta de estos aspectos, el cuestionario contempló cuatro preguntas (cfr. Castillo, Ruiz y Santiago, 2014; Ruiz, Castillo y Santiago, 2016). La primera, de tipo abierta y asociativa, se formuló para identificar los estereotipos. La segundo ofrecía una serie de encadenamientos discursivos (fundados en argumentación interna y externa) para que el estudiante determinara si dichos encadenamientos eran posibles o no, en función de sus representaciones y experiencias; en este caso se empleó una pregunta cerrada excluyente con opción múltiple. A través de la tercera pregunta se solicitó una definición de la entidad léxica tarea escolar, con miras a identificar los elementos estables de este significado y a reconstruir el significado léxico, específicamente el núcleo; aquí se utilizó, en consecuencia, una pregunta abierta. La pregunta cuatro le planteaba al participante una situación hipotética sobre la tarea escolar para que este expusiera su acuerdo o desacuerdo con ella, y así identificar, estudiar y contrastar las representaciones, a la vez que analizar la movilización

5 El cuestionario que se utilizó para la obtención de la información correspondió, en principio, al que se empleó para el caso de los docentes, definido en el proyecto DLE-361-13, en este sentido, mantuvo la misma concepción, la misma estructura y el mismo número de preguntas; en relación con estas se hicieron unos ajustes de forma y en la segunda pregunta se revisaron los encadenamientos discursivos propuestos, teniendo en cuenta para ello la edad de la población, en especial, los estudiantes de los grados sexto y séptimo. 
discursiva de valores vehiculados por la entidad léxica tarea escolar por parte de los estudiantes.

\section{Resultados: análisis y discusión}

\section{El núcleo}

Como se ha señalado, la población con la cual se adelantó el trabajo estuvo conformada por 712 estudiantes, distribuidos por ciclos así: grado 6: 1196; grado 7: 127; grado 8: 121; grado 9: 119; grado 10: 89 y grado 11: 135. Para dar cuenta del núcleo del significado léxico de tarea escolar, se recurrió a las definiciones que los estudiantes brindaron (pregunta 3 del cuestionario), caracterizados por el hecho de que los términos de base exponen una gran variedad de concepciones.
No obstante, una vez revisadas las definiciones que plantearon los estudiantes, se pudo establecer que los términos que presentaron mayores índices de frecuencia en las respuestas correspondieron a: trabajo, actividad y obligación. Estos términos tienen como complementos o finalidades las unidades léxicas: aprender, hacer, investigar, reforzar, entender, repasar, mejorar, las cuales, asimismo, conllevan la presencia de unos objetos: tema, notas, conocimiento y aprendizaje. Además de esto, también se manifiesta un locativo espacial (casa), en el que se llevan a cabo las distintas acciones que implican los términos señalados. Lo anterior se puede representar según la figura 1.

Ahora bien, desde el análisis de las definiciones propuestas por los estudiantes se estableció el

\section{TAREA}

ESCOLAR

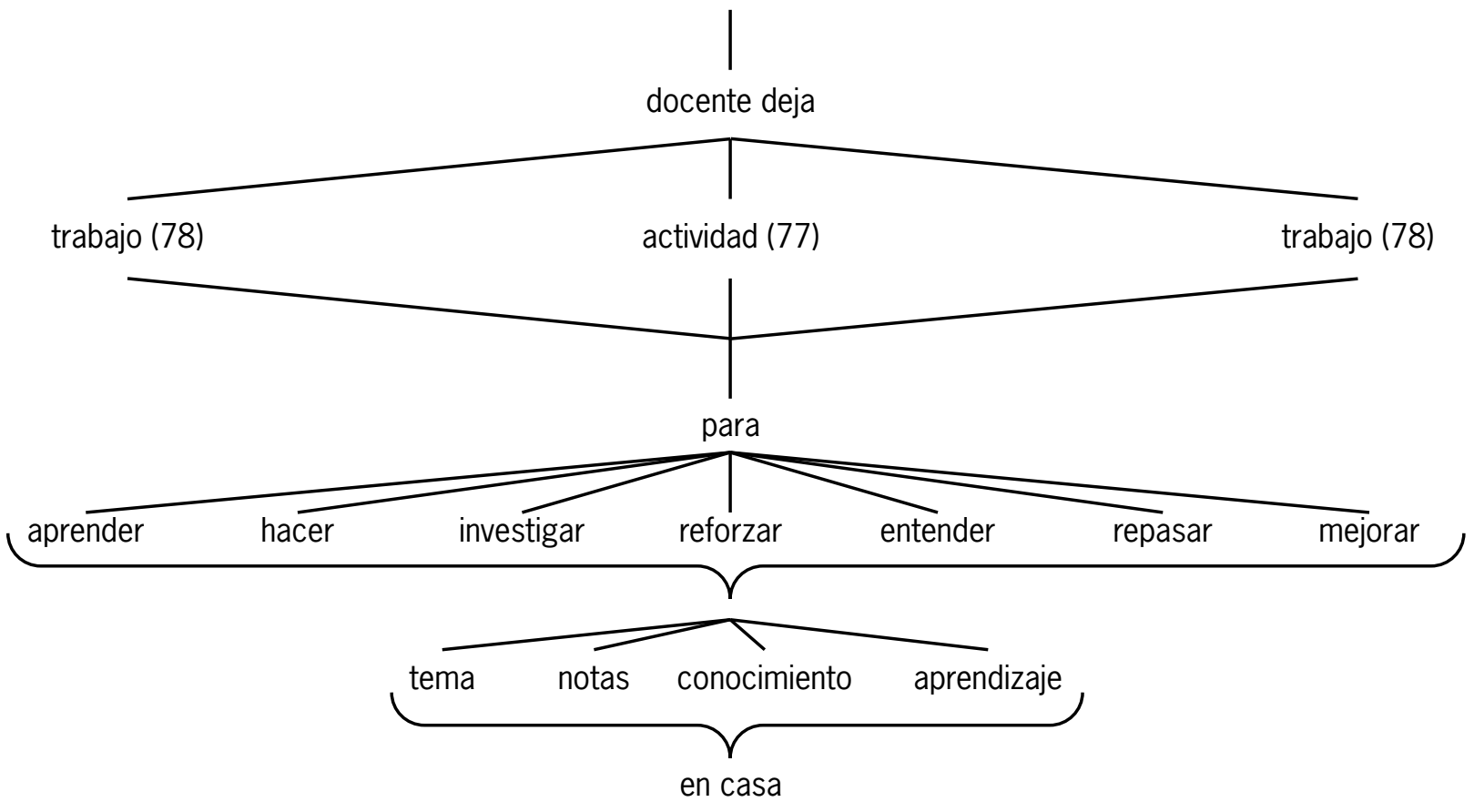

Figura 1. Definición de tarea escolar

Fuente: elaboración propia.

6 Si bien se señaló que la población para este grado fue de 120 estudiantes, solamente 119 de ellos respondieron a la pregunta que buscaba indagar por las definiciones de tarea escolar. Caso similar sucedió con el grado 11, que de los 136 respondieron 135. 
siguiente núcleo del significado de la entidad léxica en cuestión:

\section{Tarea (escolar)}

$\mathrm{N}$ : Sustantivo $\mathrm{f}$.

\section{Trabajo y actividad obligatorios en casa $\mathrm{PLT}^{7}$ Entender y aprender tema PLT Nota}

De acuerdo con esto, los estudiantes conciben la tarea como un trabajo y una actividad de carácter obligatorio que se desarrolla en la casa; este trabajo o actividad tiene como finalidad entender y aprender un tema, con lo cual se puede obtener una nota o mejorar una existente.

En cuanto al primer bloque de significado del núcleo, tanto la forma léxica trabajo como el término actividad están relacionados de forma directa, en la medida en que trabajo, como señala Moliner en su DUE (2008), se asume como la actividad que habitualmente alguien desarrolla, esto es, de lo que se ocupa en la cotidianidad; este trabajo conlleva un esfuerzo y una preparación. Actividad, por su parte, se caracteriza como el conjunto de acciones que lleva a cabo un individuo y a lo que se dedica habitualmente; las cuales remiten a una serie de operaciones de necesaria realización. En este caso, realizar las tareas es parte del quehacer cotidiano de los estudiantes; el desarrollo de estas acciones se presenta como obligatorio, en la medida en que se constituyen en actos que alguien, el estudiante, debe asumir en virtud del contrato didáctico implícito que se establece entre este y los docentes. Prima, en consecuencia, en este primer bloque, el carácter práctico que la tarea tiene para los estudiantes, dado que trabajo y actividad presuponen acción o conjunto de acciones, de ahí que la tarea es ante todo un hacer en casa.

Sobre la entidad léxica casa, se advierte en ella la diversidad de estereotipos lingüísticos que potencian la obligatoriedad de hacer algo, de efectuar una actividad en un espacio determinado. En este término aparecen como elementos esenciales el domicilio o

7 PLT corresponde, en la SPA, al conector normativo por lo tanto y, más adelante, se remite al conector transgresivo sin embargo. lugar de habitación, el conjunto de personas que en él habitan, así como las múltiples realidades y relaciones que ahí se construyen. En la medida en que este espacio particular agrupa a las personas que constituyen un núcleo familiar, permite pensar que la tarea se realiza en casa debido a que allí se puede obtener colaboración, orientación y acompañamiento, a la vez que se cuenta, en principio, con los medios necesarios para ello. La presencia de este elemento implica una suerte de desplazamiento de actividades académicas desde el aula o el establecimiento escolar hacia el hogar de los estudiantes.

El segundo bloque que configura el núcleo corresponde a la finalidad que supone la realización de la tarea. Esta se toma, entonces, como un trabajo o una actividad que una entidad -docente, profesor, escuela- le asigna a un destinatario -estudiante-, que asume que llevarla a cabo tiene como fin entender y aprender un tema o un contenido que se ha presentado (o se presentará) en el salón de clase. En términos generales, entender apunta a tener claridad sobre una temática y aprender equivale a adquirir conocimiento sobre un aspecto determinado. Según esto, hacer la tarea implica que el sujeto comprenda el significado de la temática abordada en la actividad y, a la vez, que adquiera conocimientos sobre la misma.

En ese sentido, se hace patente el carácter epistémico e intelectual de la tarea que lleva, en principio, al enriquecimiento cognitivo del estudiante. Pero también se puede señalar que priman ideas de aprendizaje bancario, en el que el estudiante recaba una serie de informaciones y datos que, a corto o mediano plazo, el docente va a recuperar mediante las actividades que impone. Este planteamiento se valida mediante la aparición, en las definiciones de los estudiantes, de tema visto, tema por ver, conocimiento, entre otras entidades léxicas.

De otro lado, el fin epistémico e intelectual de la tarea se diluye en el último bloque de significado del núcleo, en el que se presenta el carácter instrumental o pragmático de la tarea, representado en la búsqueda de resultados positivos. Realizar el trabajo permite, en última instancia, obtener una nota. El propósito último se instaura, por tanto, en 
el terreno de la valoración cuantitativa del ejercicio y lo que ello conlleva para la promoción del estudiante; así, la tarea se constituye en un medio que ayuda al tránsito de los estudiantes por los distintos niveles del sistema educativo, en esa medida se torna en un elemento útil y necesario. Según esto, prevalece el carácter pragmático de la tarea sobre los valores epistémicos e intelectuales; de esta forma, el entendimiento y el aprendizaje se supeditan, o se reducen, a la consecución de calificaciones (notas).

En este sentido, y según las definiciones aportadas por los estudiantes, se puede inferir que el aprendizaje aquí es visto de manera meramente instrumental y no se refiere a procesos cognitivos ni de transformación del individuo, sino a una simple puntuación (nota, calificación) que la tarea otorga. Vale la pena reflexionar sobre este fin último y preguntarse si esa concepción ha sido construida por el estudiante o si proviene de los discursos y prácticas tanto del sistema educativo, de los docentes, como de los padres, familiares o acudientes. Esto es, entonces, una réplica de una representación impuesta que va a marcar la vida académica del estudiante, lo que pone en evidencia una escuela en la que el aprendizaje se reduce a mera calificación.

De lo anterior se desprende la necesidad de insistir sobre aspectos fundamentales que emergen en esta concepción de tarea escolar. Se trata de las entidades léxicas aprendizaje, evaluación y calificación. Si se asume que, de manera básica, aprender/aprendizaje significa "adquirir el conocimiento de alguna cosa por medio del estudio o de la experiencia", "adquisición por la práctica de una conducta duradera”, y que evaluar corresponde a "estimar los conocimientos, actitudes y rendimientos de los estudiantes" (RAE, 2003), y calificar a "asignar una nota", en el núcleo reconstruido surgen indicadores que desvirtúan estas nociones y las prácticas que les son inherentes. Aprendizaje y evaluación corresponden, en consecuencia, a unas visiones restringidas e instrumentales que circulan en el contexto escolar y que permean no solamente las acciones escolares sino el desarrollo posterior del sujeto en el contexto académico y social.

\section{Los valores en el núcleo}

En lo que se refiere a los valores activados en los elementos del núcleo de significado de tarea escolar, en primer lugar, se encuentran los valores asociados a la zona modal axiológica (pragmáticos, intelectuales y hedónico-afectivos), luego, se hacen presentes los ontológicos (aléticos y deónticos), y por último, se ubican los pertenecientes a los de juicio de verdad (epistémicos), como se puede apreciar en la tabla 2.

En relación con los valores axiológicos, primero se ubican los pragmáticos, los cuales están en consonancia con lo señalado en relación con el núcleo y sus elementos, dado que la tarea, desde la óptica de los sujetos encuestados, es una acción útil para obtener, además de conocimientos, resultados positivos que se hacen evidentes en el aspecto cuantitativo de la evaluación, esto es, en las notas. Aspecto que, a su vez, redunda en la conquista de un objetivo mayor: aprobar la asignatura, el periodo $y$, finalmente, el año escolar. Se entrevé aquí que la tarea se asume como un medio que ayuda a obtener resultados positivos.

Tabla 2. Valores en el núcleo

\begin{tabular}{|l|l|}
\hline Epistémico & 5 \\
\hline Pragmático & 4 \\
\hline Intelectual & 4 \\
\hline Deóntico & 3 \\
\hline Alético & 3 \\
\hline Pragmático+ & 3 \\
\hline Hedónico-afectivo & 2 \\
\hline Intelectual+ & 1 \\
\hline
\end{tabular}

Fuente: elaboración propia.

Estos valores axiológicos de orden pragmático se vehiculan a través de las formas léxicas trabajo, actividad, casa y nota. Como se ha señalado, trabajo y actividad implican la acción cotidiana del individuo que tiene, en principio, una utilidad y de la que se espera un resultado. De esto se infiere que dicha práctica tiene pertinencia y que ofrece ciertos beneficios para quien la ejecuta. La nota, expresión numérica de la valoración de la que ha sido objeto el trabajo o la actividad, por parte del docente, se constituye en 
el fin de la realización de la tarea, por parte del estudiante, lo cual redundará en la aprobación satisfactoria del curso y, por tanto, en la promoción del estudiante al siguiente nivel. En este orden de ideas, la utilidad de la tarea, según la población estudiantil indagada, se relaciona con el hecho de que por medio de esta se pueden obtener calificaciones que les ayuden, no tanto para su proceso de formación, sino para la aprobación del año lectivo.

Lo intelectual, por su parte, se asocia con los lexemas trabajo, actividad, entender, aprender y tema. Se presume que tanto el trabajo como la actividad y el tema sobre el que versan son del interés del estudiante, lo cual lo motiva para desarrollar la tarea. No obstante, se debe señalar que dicho interés se torna ambivalente en la medida en que se ve afectado por el carácter obligatorio que conlleva la tarea, al ser impuesta por el docente, lo cual puede llevar a que el estudiante la encuentre interesante o no. Cumple, al respecto, desde luego, un papel central la apreciación subjetiva del estudiante, en la medida en que las temáticas que se aborden en el aula de clase se acerquen o no a sus intereses personales, elemento que incide en la condición ambivalente del valor axiológico intelectual asociado al núcleo de tarea escolar.

Entre tanto, las formas léxicas entender y aprender presuponen operaciones intelectuales de orden superior, que se constituyen en acciones de sumo interés para la configuración y el perfeccionamiento cognitivo del individuo. El hecho de comprender una temática e integrar el conocimiento que de ahí se deriva en los esquemas mentales del individuo -lo cual, desde una perspectiva cognitiva, conlleva aprendizaje- es, sin duda, un elemento de interés intelectual, de ahí la presencia de este valor en las consideraciones de los encuestados en torno a la tarea escolar. Pese a ello, como se ha expuesto, esto se ve opacado por el fin último: obtener una buena calificación.

Ahora bien, si la tarea surge del convenio implícito que se da entre los actores principales del proceso educativo, docente y estudiante, esta se puede asumir, entonces, como una norma socioeducativa, de ahí la presencia de los valores ontológicos deónticos que se presentan en el núcleo. El trabajo o la actividad, al formar parte de las actividades académicas cotidianas de los estudiantes, se tornan en un compromiso

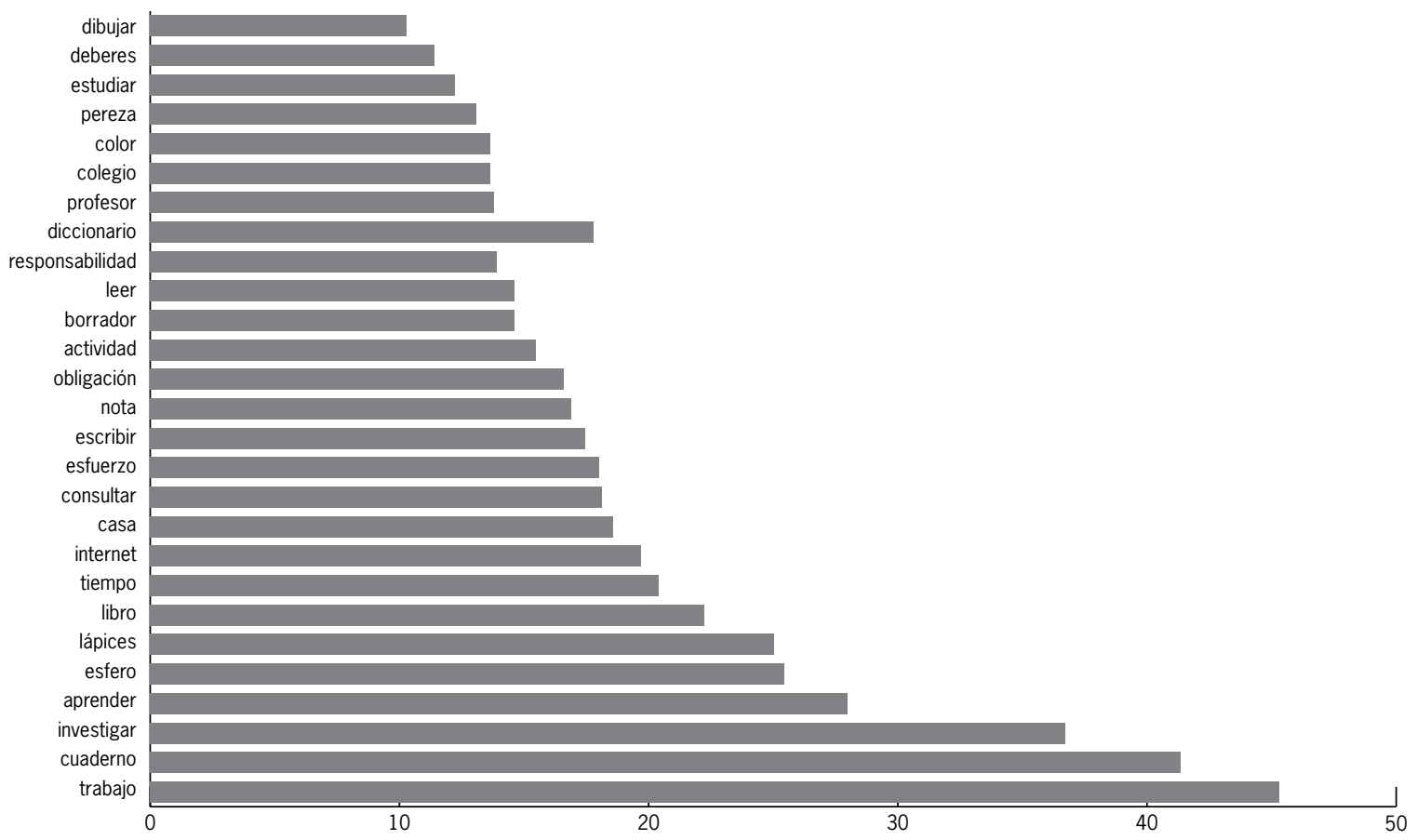

Figura 2. Distribución porcentual de los estereotipos

Fuente: elaboración propia. 
que estos adquieren y que se vuelve de imperativo cumplimiento. Esta presencia de lo obligatorio en el núcleo del significado de tarea escolar se hace evidente, precisamente, en la forma léxica obligación.

Por último, y sin ser significativo su número, unos valores de la zona modal axiológica imprimen al núcleo la doble posibilidad de lo agradable/desagradable. Se trata de los valores hedónico-afectivos que se activan en razón a la presencia de los elementos casa y nota: la ambivalencia se establece por lo que este espacio y este resultado, o fin último, pueden generar en el cumplimiento de la tarea escolar. El aporte positivo de las relaciones y de los medios encontrados en el hogar, así como la obtención de una nota significativa generará sentimientos positivos en el sujeto; caso contrario sucederá, si no se dan estas condiciones contextuales o no se alcanza la calificación esperada o necesitada.

\section{Los estereotipos}

Como se señaló en la fundamentación teórica, los estereotipos de una entidad léxica dan cuenta de las representaciones lingüísticas que un individuo o una comunidad tienen de esa dicha entidad y se presentan como un conjunto abierto. A partir del análisis de los datos, específicamente de la primera pregunta del cuestionario, se identificó un total de 1000 palabras asociadas a la entidad léxica tarea escolar. De este número global de estereotipos, muy variado y extenso, se pudo determinar que 27 términos aparecen como los más frecuentes y los más representativos, de manera que se erigen como el conjunto de asociaciones que ofreció la población indagada y que dan cuenta de la representación que tiene de la tarea. En la figura 2 se presenta la distribución porcentual de dichas unidades léxicas.

Como se puede apreciar, los términos que los estudiantes asocian con tarea escolar hacen referencia a diversidad de aspectos que involucran acciones, sean estas de orden físico o cognitivo (trabajo, investigar, aprender, consultar, escribir, actividad, leer, estudiar, dibujar), instrumentos con los cuales adelantarla (cuaderno, esfero, lápices, libro, internet, borrador, diccionario, colores), relaciones frente a esta (esfuerzo, obligación, responsabilidad, pereza, deber) y otros concernientes con el lugar (casa, colegio), el tiempo, el generador de la tarea (profesor) y la calificación de la misma (nota).

Se evidencian en estas formas léxicas los elementos que, desde la visión de los estudiantes, son relevantes y se hacen presentes al momento de caracterizar y asumir la noción de tarea; no obstante, de estos se hacen notorios los que tienen que ver con acciones (nueve formas léxicas) enseguida se encuentran los referidos a los instrumentos que posibilitan la ejecución de la tarea (ocho formas léxicas) y en tercer lugar se destacan los asociados a las relaciones de los estudiantes frente a la tarea (cinco formas léxicas).

De otra parte, los estereotipos identificados en las respuestas de los estudiantes se caracterizan por tres aspectos básicos: 1) retoman y ratifican los elementos del núcleo; 2 ) incluyen nuevas asociaciones que despliegan algunos elementos y valores del núcleo;3) algunos términos de este conjunto abierto dan cuenta de entidades concretas, de objetos propios del mundo escolar en los que pueden desarrollarse las tareas, $o$ con ayuda de ellos, pero que no son las tareas en sí.

Si se asume la agrupación de los estereotipos en función de los aspectos que implica la tarea, hay que considerar, en primera instancia, los elementos que remiten a acciones. En esta se ubican los términos: trabajo, investigar, aprender, consultar, escribir, actividad, leer, estudiar, dibujar. Dentro de este grupo de formas léxicas, la que más frecuencia presenta es trabajo, que corresponde, precisamente, a un elemento del primer bloque del núcleo de significado de tarea escolar. La existencia de trabajo, tanto en el núcleo como en el conjunto de estereotipos, da cuenta de la reiteración de la idea presente en los elementos estables y en las asociaciones, esto es, que la tarea es un trabajo. En este sentido, remite a las acciones que el estudiante debe realizar en función de lo que el profesor impone.

Asimismo, la entidad léxica actividad retoma y desarrolla la representación básica (núcleo) de la tarea en las asociaciones frecuentes y más importantes del grupo de estudiantes. Como en el caso 
del término anterior, la palabra actividad pone de manifiesto la serie de acciones, tanto físicas como mentales, que debe ejecutar el estudiante con miras a cumplir con la tarea escolar.

Es interesante la consolidación de estas dos entidades léxicas entre los estereotipos presentes en el núcleo, en la medida en que los estudiantes insisten en el hacer como componente fundamental de la tarea o como la propia tarea. Esta actividad se concreta, en la vida escolar, en otros términos, que aparecen en las concepciones de los estudiantes. Estas palabras actualizan tales trabajos y actividades, pues remiten a objetos en los que se puede consignar el fruto de la acción efectuada o con los que esta se desarrolla; dichos términos son: cuaderno, esfero, lápices, borrador y colores.

Ahora bien, estos términos referidos a objetos se declinan en dos grandes grupos: por una parte, los útiles escolares con -y en- los que los informantes realizan las acciones impuestas; por otra parte, los recursos que proveen los contenidos, las informaciones, así como los datos que el estudiante debe recuperar mediante un hacer para transcribirlos; se trata de los recursos representados por los términos libro, internet y diccionario.

A pesar de la presencia del término aprender en los estereotipos, es necesario preguntar si esta operación cognitiva es el propósito tanto del docente como del estudiante. Lo anterior dado que el papel de los útiles escolares en las representaciones de los estudiantes es muy importante, ello debido tal vez al hecho de que por medio de estos objetos los estudiantes pueden reflejar o demostrar su trabajo, sus actividades.

De este conjunto de objetos, de materiales o de útiles escolares, el término que presenta el mayor número de ocurrencias corresponde a cuaderno (294 oc.), que de hecho ocupa la segunda posición en el listado general de los estereotipos, lo cual muestra que dicho instrumento tiene gran importancia para el estudiante en la ejecución de la tarea, pues es en este en donde, por lo general, registra y da cuenta de esta, además de lo que se presenta en el aula de clases; asimismo, se constituye en objeto de revisión y evaluación por parte del docente, de tal forma que se erige en fuente de notas. Ahora bien, para llevar a cabo dicho registro es necesario otro elemento: el esfero, que aparece en segundo lugar en este subconjunto (181 oc.) y en el quinto del listado general.

Otros términos contribuyen a aclarar o, mejor, a esgrimir la siguiente hipótesis: se requieren una serie de acciones elementales para cambiar el depósito de la información, es decir, transcribir los datos de los libros o de internet a los cuadernos. Algunos otros términos como leer y escribir hacen visibles estas acciones, esta serie de actividades cuya huella se plasma en las hojas de un cuaderno.

A los dos términos anteriores se deben agregar las formas léxicas investigar, consultar, estudiar y dibujar como realizaciones de la actividad. En principio, consultar, estudiar e investigar se presentan como acciones que implican operaciones cognitivas de orden superior que toman forma en leer y escribir, pero contienen una serie de representaciones, al menos investigar, que probablemente provienen del discurso de los docentes y se han instalado en las creencias de los estudiantes. Según el DUE (Moliner, 2008), investigar se define como "estudiar o trabajar en un campo del saber para aumentar los conocimientos sobre una determinada materia", y entonces se podría asumir que el docente recurre a esta concepción de investigar/investigación, pero no deja ver su claridad con respecto a esta actividad académica como profesor que posee una formación de base, la experiencia y la formación permanente.

Ahora bien, aunque las actividades investigar y consultar refieren en el ámbito académico a la resolución de situaciones problemáticas, lo que lleva a la comprensión de estas y, con ello, a la consecución de conocimiento, en especial lo referido a investigar, en el contexto escolar esta actividad se reduce a la consecución de información (datos, definiciones, ejemplos, etc.), a través de un medio (libro, diccionario o internet), que generalmente se consigna en el cuaderno.

Por su parte, consultar, estudiar y dibujar traducen quizá de manera más evidente y clara lo que realiza el estudiante como actividad, pero se mantienen los 
dos primeros términos, como reiteraciones de leer y escribir, sin que ello signifique una actividad más exigente en el plano cognitivo y social. Según las respuestas de los estudiantes, investigar, consultar, estudiar y dibujar son aspectos que suponen la adquisición y el uso de conocimientos. En este sentido, estos estereotipos se asocian con los dos primeros bloques del núcleo que tienen que ver con trabajo, actividad, entender y aprender.

Con respecto a la presencia de los lexemas profesor y colegio, se puede afirmar que remiten a la idea de que este actor educativo y esta institución social son los que generan e imponen las tareas escolares. La forma léxica colegio es el hiperónimo y corresponde a la instancia que tiene como función social el proceso de formación del individuo. Como parte del quehacer de la institución educativa se ha instaurado la tarea escolar en sus actividades formativas, es en el colegio, entonces, donde se concibe, se revisa, se valora y, especialmente, se califica la tarea, de ahí que los estudiantes incluyan esta palabra dentro de las asociaciones. Por su parte, el profesor, hipónimo, al formar parte de esta institución, es el profesional encargado de llevar a la práctica cotidiana la formulación de la tarea, en esa medida, es el actor educativo que origina e impone la tarea como trabajo o actividad para que el estudiante la desarrolle de manera obligatoria en casa. De esta forma, en la relación que se establece entre profesor/estudiante/ contenidos, la tarea se instaura como uno de los recursos que permite actualizar dicha relación.

Un aspecto importante en esta representación de la tarea escolar como actor/institución es la ausencia de un término que dé cuente del estudiante. Por esta razón, la palabra profesor cobra mayor fuerza, pues se ha vuelto fundamental en estas asociaciones de la tarea. Se está autorizado, entonces, a considerar que el docente representa la autoridad que impone el trabajo que el estudiante ha de ejecutar. Priman, por tanto, las acciones por desarrollar como transformación de los términos actividad y trabajo, y se desvanece toda asociación con el sujeto que debe encargarse de su realización. Además, como se verá más adelante, mucho más claro que la acción del sujeto sin designación entre los estereotipos es lo referido al propósito último de la actividad escolar que, en términos de los informantes, corresponde a la nota, como se ha señalado. Está ausente, entonces, la representación del sujeto, pero permanece la voluntad que se orienta hacia una consecución pragmática.

En estrecha relación con la presencia-ausencia de designaciones de los actores educativos se identifican los términos obligación, esfuerzo, pereza y responsabilidad. La forma léxica obligación, que se complementa con la aparición del término deber, ratifica el hecho de que el estudiante se ve, o está, forzado a hacer la tarea, en virtud de la forma en que se desenvuelve la situación didáctica, en la que, como los estudiantes señalan, el profesor les impone ciertas actividades, una de ellas, la tarea escolar. Esta imposición genera, desde luego, reacciones o actitudes en los discentes, que en los estereotipos se manifiestan con los términos: esfuerzo, responsabilidad y pereza.

El profesor, legitimado por su autoridad, se asocia con obligación que se traduce en "hacer que alguien realice cierta cosa usando para ello la ${ }^{\star}$ fuerza o la ${ }^{\star} a u-$ toridad" (Moliner, 2008). Frente a esta decisión del docente, se ve surgir en el estudiante una serie de representaciones que dan cuenta de su relación con la tarea, pues en ningún momento los estereotipos revelan reacción hacia/contra el maestro o su autoridad.

Por tanto, si la tarea se entiende, con base en lo señalado, como trabajo o actividad obligatoria, la presencia de la forma esfuerzo es coherente, en la medida en que el trabajo o la actividad requiere que el individuo se obligue a desarrollarla y en ello invierta recursos físicos, intelectuales y hasta económicos. Este esfuerzo se posiciona como una condición necesaria para desarrollar las actividades establecidas de manera que su logro facilite la obtención de una calificación, propósito final de esta actividad educativa. Al respecto, es necesario preguntarse si los aspectos de orden negativo que conlleva este esfuerzo, como cansancio, fatiga, estrés, aburrimiento, podrían convertirse en las justificaciones de la búsqueda de la nota como resultado de la tarea. 
Asimismo, en una relación de oposición con la palabra esfuerzo, los informantes activan en sus representaciones el término pereza. Esta forma léxica, que indica desgano y falta de voluntad frente al trabajo o la actividad asignadas, permite proponer una doble posibilidad de comprensión: contra la pereza frente a una actividad impuesta se debe asegurar un esfuerzo para superarla o, tal vez, es obligatorio un esfuerzo para realizarla, todo ello con el fin de alcanzar un buen resultado en términos cuantitativos.

A pesar de lo expuesto, la obligación y el esfuerzo posibilitan, asimismo, que el estudiante vea aspectos positivos en el desarrollo de la tarea, en especial que adquiera responsabilidad. La presencia de este término en los estereotipos señala el hecho de que el estudiante, gracias a la tarea, es consciente de sus obligaciones y, asimismo, actúa de forma adecuada a las exigencias que le impone la realidad y el contexto académico. Esto supondría, sin hacerse evidente ni en el núcleo ni en los estereotipos, el desarrollo de autonomía; sin embargo, la fuerza que trae consigo la obligatoriedad le resta valor y preponderancia a dicho desarrollo.

Aunque se puede establecer una argumentación del tipo tarea escolar PLT obligación PLT pereza SE responsabilidad PLT esfuerzo, se puede determinar que esta serie de relaciones, que está acorde con el protocolo del significado léxico, se modifica con la aparición de una entidad léxica que revela la finalidad y la voluntad que dan fortaleza a una orientación utilitarista de la tarea escolar. Esta representación de la tarea corresponde al término nota, objetivo de la tarea, como ya se ha señalado.

En este orden de ideas, el carácter pragmático que tiene la tarea, según las representaciones de los estudiantes, además de estar presente en los subconjuntos acciones e instrumentos previamente analizados, se refuerza con la presencia de la forma léxica nota en los estereotipos. Como se ha argumentado, el fin último de la tarea es la obtención de calificaciones que permitan la certificación positiva del estudiante y su promoción, lo cual se expresa, en el ámbito escolar, por medio de la nota; en ese sentido, este elemento, para un grupo de estudiantes, es necesario y es el que alienta hacer las tareas. Según esto, la satisfacción que puede generar el hacer las tareas no se asocia con el crecimiento cognitivo o el aprendizaje sino con la obtención de una buena calificación. Esto permite inferir una concepción cuantitativa y punitiva de la evaluación, centrada en el producto y no en el proceso.

Cabe preguntar en este punto si la tarea asociada a la nota, tarea escolar PLT nota, en una relación de medio/fin, resulta de las experiencias educativas de los estudiantes, de las prácticas escolares, del discurso de los profesores o de las discusiones en familia. En todo caso, la representación de la tarea se distorsiona y se reduce a la obtención de premios o a evitar castigos, lejos está una concepción de tarea escolar que recupere el valor del sujeto que aprende y sus desarrollos personales y sociales para la vida sociocultural.

Sin embargo, la obtención de un resultado positivo requiere implicarse y prever una serie de condiciones. Son estos factores los que se establecerán como la base, como el medio, entre otros ya enunciados, para el logro previsto. Se trata aquí de la dimensión espacio-temporal, presente en el núcleo reconstruido y en los estereotipos de tarea escolar: casa y tiempo.

Entonces, la tarea escolar conlleva una locación espacial específica para su elaboración: la casa del estudiante. Desde esta perspectiva, la tarea se debe hacer fuera del aula de clase, es más, fuera de la institución escolar y el lugar más adecuado para ello es la vivienda del estudiante. Esto supone, por un lado, trasladar el aula a la casa y, del otro, la participación de sus habitantes en dicha elaboración, como se expuso párrafos atrás.

La palabra casa, con una presencia reiterada en las asociaciones pues aparece en el núcleo reconstruido, introduce en este conjunto amplio de representaciones de la tarea escolar una variedad de actores que no siempre poseen las bases suficientes para apoyar, orientar ni apreciar las actividades que el estudiante lleva a su hogar como asignación del docente. En este sentido, este espacio vital se constituye en una proyección del colegio, o del aula de clase, sin que el estudiante disponga de elementos 
indispensables como los que podría compartir en el espacio escolar para desarrollar trabajos y ejercicios con miras a desarrollar su individualidad y su carácter social. Las explicaciones del docente, el cuestionamiento, los talleres, la interacción estudiante/estudiante o estudiante/docente, algunos recursos documentales, entre otros, ya no podrán estar a disposición del estudiante cuando se encuentre en su hogar.

Si bien algunas actividades podrían develar procesos, dificultades, fortalezas, etc., al realizarlas en su casa, en variadas ocasiones lo que ha de hacer el estudiante en su casa, con o sin ayuda, responde a una necesidad de completar contenidos, de tratar temáticas para cuyo desarrollo el tiempo en el aula fue insuficiente, quizá por los avatares mismos de la escuela. ¿Acaso es esta la mejor manera de contribuir a una educación genuina?

En el párrafo anterior se evidencia la otra dimensión necesaria en el desarrollo de la tarea escolar y se trata del término tiempo. La presencia de tiempo se puede explicar por el hecho de que la realización del trabajo o actividad implica, de acuerdo con lo expuesto, obligación y esfuerzo, supone, además, la inversión de tiempo para su ejecución y su culminación, de ahí que el tiempo sea una condición necesaria. Así, los estudiantes asocian a tarea escolar el término tiempo dado que deben dedicar parte de su tiempo libre al desarrollo de la tarea. ¿Tiempo insuficiente en la jornada escolar? $¿$ Tiempo perdido? ¿Tiempo libre? ¿Tiempo indispensable para la realización de una actividad? He aquí una serie de preguntas que glosan la representación de tiempo en la tarea escolar. Estas variantes de tiempo significan quizá toda la gama de opciones en que se traduce la idea de tiempo. El estudiante asume probablemente que, ante la insuficiencia de tiempo en las acciones escolares, dependientes del colegio y del profesor, para estudiar todos los temas y para desarrollar todas las actividades previstas, la autoridad educativa recurre a su tiempo libre para imponerle una serie de trabajos por desarrollar en su casa para culminar el programa del curso.
Sin embargo, otra opción es posible y concierne a evitar perder el tiempo. Al ocuparse, al ocupar el tiempo de no asistencia al colegio, el estudiante no tomará caminos indebidos en la calle ni con amistades poco recomendables. En este orden de ideas, invertirá su tiempo libre en actividades provechosas y, en teoría, formativas.

\section{Los valores en los estereotipos}

Las nuevas asociaciones, emergentes en las respuestas de los estudiantes, no solamente refuerzan y amplían los elementos del núcleo, el lexicográfico y el reconstruido, sino que introducen una consolidación de los valores. En la figura 3 se presenta la distribución de los valores presentes en los estereotipos.

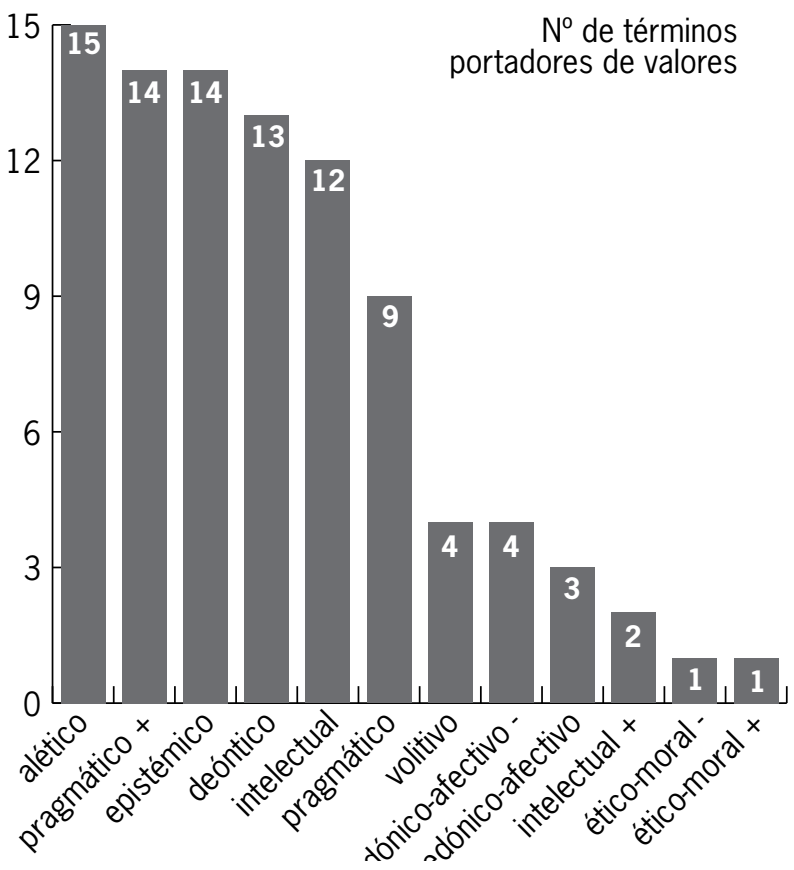

Figura 3. Valores en los estereotipos

Fuente: elaboración propia.

En primer lugar, se destacan los valores pragmáticos, pertenecientes a la zona modal axiológica, dado que las acciones que la tarea supone son útiles en la medida en que, de acuerdo con lo señalado en los párrafos anteriores, viabilizan el aprendizaje y, en consecuencia, permiten obtener resultados que se reflejan en la nota y en la promoción del estudiante. Ahora bien, las actividades que implican la realización de la tarea involucran operaciones de 
orden cognitivo que posibilitan obtener conocimientos acerca de las diversas temáticas que se desarrollan en la escuela, de ahí la alta presencia de valores epistémicos e intelectuales. Estos valores se actualizan bajo las formas léxicas como leer, que se puede entender como actividad intelectual que tiene como fin la comprensión de un texto, con lo cual se consigue, asimismo, tener conocimiento sobre la temática que aquel desarrolla, lo que lleva a que el individuo enriquezca y reestructure sus esquemas mentales, esto es, a que aprenda. Aprender, desde la perspectiva planteada, se entiende como la adquisición de conocimientos de diversa índole y consolida los valores contenidos en los elementos del núcleo (epistémicos y pragmáticos).

Si bien los valores pragmáticos, epistémicos e intelectuales son los que priman en este conjunto de formas léxicas, también están presentes los valores aléticos y deónticos debido al carácter necesario y obligatorio que tiene la ejecución de la tarea. Con esta activación de valores en los estereotipos se refuerzan los valores contenidos en algunos elementos del núcleo. Estos valores se reafirman en, y con, las palabras investigar y consultar que se tornan, tal y como se ha señalado, en acciones necesarias y obligatorias para cumplir con los trabajos y actividades que se le asignan al estudiante.

Los valores pragmáticos son más reiterativos en otros términos asociados a la tarea escolar como cuaderno, esfero, lápices, libro, borrador, diccionario y colores. Dada la utilidad que presentan estos recursos (útiles escolares), en esta subcategoría priman los valores ligados a la utilidad/inutilidad, lo cual ratifica el carácter práctico que de la tarea escolar se decanta de las respuestas de los estudiantes indagados. La alta presencia de las formas léxicas ligadas a estos útiles escolares deja entrever que para los estudiantes tienen gran relevancia los medios con los cuales se desarrolla la actividad propuesta, de tal forma que, además de los valores pragmáticos, se evidencian los deónticos y los aléticos en este subgrupo de estereotipos.

Lo anterior implica que dada la conveniencia que tienen, los útiles se tornan en elementos necesarios y obligatorios en el contexto escolar, sin los cuales difícilmente se podrían adelantar las distintas actividades que implica la cotidianidad en la escuela. En esa medida, estos medios se asumen como provechosos, prácticos y eficaces para llevar a cabo una actividad beneficiosa, en este caso, la tarea; en la medida en que aporta aprendizaje, tal y como es entendido por los estudiantes, esto es, la obtención de notas.

Como se expuso antes, si se tiene en cuenta que la tarea favorece el aprender y entender un tema y que para ello son necesarios unos insumos, en este subconjunto hacen presencia, en menor medida, los valores epistémicos e intelectuales, asociados especialmente a las formas léxicas libro, internet y diccionario. Libro y diccionario forman parte de los medios en la medida en que gracias a estos se pueden, en principio, adelantar las indagaciones y las consultas que suponen las actividades y los trabajos impuestos en el colegio, de manera que se configuran como fuentes de información y de acceso al conocimiento. También se convierte en fuente de consulta internet, que no forma parte de los útiles pero que sí es un recurso al que pueden acceder, en teoría, todos los estudiantes para conseguir información. Así las cosas, libro, diccionario e internet permiten tener acceso a informaciones que facilitan el adelantar la tarea y resultan, en consecuencia, en aprendizaje y entendimiento.

La orientación deóntica en las concepciones de los estudiantes se refuerza con la idea de obligatoriedad que el grupo de informantes activa, y que ya aparecía en el núcleo, con los términos obligación y deberes. Este tipo de valores, contenido también en el término esfuerzo, indispensable para realizar una actividad o un trabajo, propicia la aparición de valores en la zona modal axiológica, específicamente los valores hedónicos-afectivos y ético-morales negativos. Estos valores están presentes en las formas cansancio, fatiga, estrés y aburrimiento.

La concepción negativa de la tarea escolar se evidencia de nuevo con la activación, en otros términos, de los valores hedónicos-afectivos, relacionados con el tiempo y su uso. En este orden de ideas, el 
estudiante asume como un sacrificio el recurrir a su tiempo libre para ejecutar las actividades asignadas. Frente a esta dimensión negativa, aparece una idea positiva representada en la responsabilidad necesaria para el cumplimiento de tales actividades. Se trata en este caso del refuerzo de valores ético-morales de orden positivo mediante la asociación de tarea escolar con la palabra responsabilidad.

\section{Consideraciones finales}

El estudio reveló que los elementos estables, o esenciales del significado de tarea escolar, desde la perspectiva de los estudiantes, corresponden a trabajo, actividad, obligatorio, casa, entender y aprender. De esta forma, la idea básica de tarea escolar, expuesta en Castillo, Santiago y Ruiz (2014) y que surge del discurso lexicográfico, Obra y trabajo obligatorios PLT Dificultad PLT Esfuerzo SE Tiempo limitado PLT Resultado, se actualiza en Trabajo y actividad obligatorios en casa PLT Entender y aprender tema PLT Nota.

Estas nuevas entidades léxicas en el núcleo develan que el estudiante sitúa la realización de las actividades, propias de la tarea, en la casa, lo que lleva a deducir que se restringe el espacio de la ejecución de la tarea y se desplaza la responsabilidad en el acompañamiento a la familia (padres, acudientes y otros familiares).

Por otra parte, a diferencia del núcleo lexicográfico, en los elementos esenciales de la definición de los estudiantes aparecen los términos entender y aprender un tema. En principio, estas formas léxicas se circunscriben al contexto escolar y se reducen a las actividades cognitivas que llevan a facilitar la memorización de contenidos, de datos, representados en la palabra tema.

En el discurso de los estudiantes hay una gran variedad de asociaciones cuando se trata de relacionar la expresión tarea escolar con otros términos. Un número muy amplio de representaciones aparece asociado a esta entidad léxica, pero fundamentalmente 27 son los términos representativos en razón a sus ocurrencias. Además, de estas 27 palabras recurrentes en las concepciones que los estudiantes tienen de tarea escolar, solamente cinco (5) consolidan los estereotipos lexicográficos, es decir, el conjunto amplio de elementos lingüísticos de base que socialmente se han generado y que se recogen en los diccionarios que se han tomado como referencia. Veintidós (22) términos realmente diferentes se activan en las respuestas de los estudiantes. No solo se puede establecer, con estas características, una gran variedad y dispersión de visiones con respecto a las tareas, como resultado de diversas voces y prácticas influyentes, sino también la asociación preponderante con útiles escolares, con medios y recursos. Esta primacía de los objetos, en detrimento de las acciones centradas en lo cognitivo, pone de manifiesto la idea de la tarea vinculada fuertemente a los materiales utilizados para desarrollarla. Este aspecto parece de importancia para las reflexiones tanto de docentes como de padres de familia. Otro aspecto que resalta corresponde a la ausencia, entre las representaciones más frecuentes, de asociaciones con el campo de la formación, del desarrollo personal y social.

Contrasta con estos debilitamientos, tanto de los elementos del núcleo como de los estereotipos, la función que se le asigna a la tarea, pues entre las nuevas asociaciones predomina una visión utilitarista de esta. De ahí que su función es servir como base o medio para alcanzar notas. Esta idea se refuerza y se despliega, junto con los valores portados, en el conjunto abierto de asociaciones establecidas con el término tarea y que ya aparecía en el último bloque de significado del núcleo reconstruido. Prima, por tanto, una orientación pragmática positiva sobre otros valores sociales complejos, quizá más urgentes e indispensables, en tiempos como los que hoy viven los colombianos, tales como los ético-morales, los intelectuales y otros de la zona modal de juicios de verdad (epistémicos). Con el predominio de estas dimensiones utilitaristas se da cuenta de un fenómeno inquietante: la distorsión de la función y del sentido de la educación, en general, y de la tarea escolar, en particular. Esto tiene incidencias en la vida familiar, las cuales se incrementan posteriormente en la vida social, pues se trata de una acción estructurada, desarrollada con arreglo a fines, en la que el medio se consolida en la tarea y en los aprendizajes (aparentes). 
La investigación contribuyó a identificar, entre 712 estudiantes de la ciudad de Bogotá, un alto número de estereotipos que se relacionan con la palabra tarea (escolar). Asimismo, a partir de dichas formas modales, se logró caracterizar el grupo de asociaciones que, como conjunto abierto, evidencia la diversidad de concepciones de tarea (escolar) que registran los encuestados. El estudio de los valores activados en los estereotipos permitió determinar las orientaciones axiológicas de los informantes y proponer algunas hipótesis que explican cómo las representaciones, presentes en los discursos, provienen de diversas influencias del contexto escolar.

Por último, en relación con los objetivos previstos, se puede considerar que, en sus discursos, los estudiantes movilizan nuevas y variadas asociaciones que establecen con la forma léxica tarea escolar. A pesar de los numerosos términos nuevos, no se logra determinar una unidad que los integre en una visión coherente. En relación con los valores portados por la diversidad de asociaciones se concluye que priman dos tipos de valores en la zona modal axiológica y uno propio de los valores de juicios de verdad. En los primeros, se destacan los valores pragmáticos positivos, orientados especialmente al logro de resultados representados en una nota, entonces meramente instrumentales, y los intelectuales positivos que dan cuenta del interés, la motivación, generados en los estudiantes por los temas que son objeto de las tareas. Ahora bien, estos valores intelectuales se relacionan estrechamente con los epistémicos, activados justamente en relación con los contenidos, los saberes declarativos, las informaciones a los que se reducen generalmente las tareas escolares. En este sentido, la orientación tanto epistémica como la intelectual se desdibuja en el afán de la obtención de una calificación, esto es, de un número que, aparentemente, representa los múltiples aprendizajes que se logran en el contexto escolar.

Lo identificado y expuesto revela diversas problemáticas que habitan en el mundo escolar y que exigen una pronta y sólida intervención para su transformación. Tanto maestros como estudiantes, padres de familia, acudientes y el sistema educativo mismo se encuentran inmersos en un serio impasse que solamente el estudio crítico, la formación/actualización y las propuestas innovadoras responsables y sólidas podrán contribuir a resolver.

\section{Referencias}

Castillo, M., Santiago, G. y Ruiz, J. (2014). La representación del significado léxico y la movilización de valores sociales: la tarea escolar. Folios, 40, 105-124.

Dervin, F. (2009). Approches dialogiques et énonciatives de l'interculturel : pour une didactique des langues et de l'identité mouvante des sujets. Synergies Roumanie, 4, 165-178.

Galatanu, O. (1999). Le phénomène sémantico-discursif de déconstruction-reconstruction des topoï dans une sémantique argumentative intégrée. Langue française, 123, 41-51.

Galatanu, O. (2000). Signification, sens et construction discursive de soi et du monde. En J.M. Barbier y O. Galatanu (eds.), Signification, sens, formation (pp. 25-43). París: PUF.

Galatanu, O. (2002). Le concept de modalité: Les valeurs dans la langue et dans le discours. En O. Galatanu y F. Le Roy (eds.), Les valeurs, séminaire "le lien social" (pp. 17-32). Nantes: Maison des Sciences de l'Homme.

Galatanu O. (2003a). La sémantique des possibles argumentatifs et ses enjeux pour l'analyse du discours. En I. Iñarrea Las Heras y M. J. Salinero Cascantel (coord.), El texto como encrucijada: estudios franceses y francófonos (pp. 213-226). Logroño: Universidad de la Rioja.

Galatanu, O. (2003b). La construction discursive des valeurs. En J.M. Barbier (eds.), Valeurs et activités professionnelles (pp. 87-114.). Séminaire du Centre de Recherche sur la Formation du CNAM. París: L'Harmattan.

Galatanu, O. (2005). Analyse du discours. Diversité Ville, école, intégration, 140, 55-61. Recuperado de http:// www.cndp.fr/archivage/valid/70174/70174-1077813726.pdf

Galatanu, O. (2006). Le cinétisme de la signification lexicale. En J.M. Barbier y M. Durand (eds.), Sujets, activité, environnement (pp. 85-104). París: Presses Universitaires de France.

Galatanu, O. (2007). Sémantique des possibles argumentatifs et axiologisation discursive. En D. Bouchard y 
J. Evrard (eds.), Représentation du sens linguistique 2 (pp. 313-325). Louvain-La-Neuve: Duculot De Boeck.

Galatanu, O. (2009). Les incidences sémantiques des déploiements argumentatifs dépendants du co-(n) texte de production du discours. En E. Havu, J. Härmä, M. Helkkula, M. Larjavaara, U. Tuomarla (eds.), Mémoires de la Société Néophilologique de Helsinki LXXVIII. La langue en contexte. Actes du colloque Représentations du sens linguistique IV, (pp. 391-404). Helsinki : Société Néophilologique.

Moliner, M. (2008). Diccionario de uso del español. Madrid: Gredos.
Putnam, H. (1975). The meaning of "meaning". En K. Gunderson (dir.), Language, mind and knowledge (pp. 131-193). Minnesota: Minnesota Press.

Real Academia Española (RAE) (2003). Diccionario de la lengua española. 22a. ed. Madrid: Espasa Calpe.

Ruiz, J. (2013). Des interfaces pour l'analyse de la signification et du sens. Folios, 37, 27-50.

Ruiz, J., Castillo, M. y Santiago, W. (2015). Las tareas escolares: representaciones de los docentes de lenguas. Folios, 42, 105-126.

Ruiz, J., Castillo, M. y Santiago, W. (2016). Aproximación la representación del significado léxico de la entidad léxica tarea escolar. Forma y Función, 29(1), 151-177.

Para citar este artículo:

Ruiz Vega, J., Santiago Galvis, A. W. y Castillo Perilla, M. (2018). La tarea escolar: representaciones lingüísticas de los estudiantes. Folios, 48, 101-119. 